\title{
A First-Order DPA Attack Against AES in Counter Mode with Unknown Initial Counter
}

\author{
Josh Jaffe \\ Cryptography Research, Inc. \\ 575 Market Street, suite 2150, San Francisco, CA 94105, USA
}

josh@cryptography.com

\begin{abstract}
Previous first-order differential power analysis (DPA) attacks have depended on knowledge of the target algorithm's input or output. This paper describes a first-order DPA attack against AES in counter mode, in which the initial counter and output values are all unknown.
\end{abstract}

Keywords: power analysis, SPA, DPA, HO-DPA, AES, counter mode.

\section{Introduction}

Previous first-order differential power analysis (DPA) attacks have depended on knowledge of the target algorithm's input or output 1] 2]. This paper describes a first-order DPA attack against the Advanced Encryption Standard (AES) 3] in counter mode, in which the initial counter, input values, and output values are all unknown.

The attack proceeds as follows. Suppose the input data to an algorithm is unknown, but can be expressed as single secret constant summed with known, variable data. The known, variable part of the data is used to mount a DPA attack, and the secret constant is treated as part of the key to be recovered. The "key" recovered by the DPA attack is then a function of the actual key and the secret constant. The known input values are then combined with the recovered "key" to compute the actual intermediate values produced by the algorithm. The recovered intermediates are then used to carry the attack forward into later rounds, enabling additional DPA attacks to recover the real key.

The attack also addresses the challenges to DPA presented by block ciphers used in counter mode 4. DPA attacks target secrets when they are mixed with known variable quantities. In counter mode only the low-order bits of the input change with each encryption. Hence there are few variable intermediates to target in the first round of a typical block cipher. We demonstrate a method for propagating the attack into later rounds in which more known, variable data is available.

Although counter mode presents additional challenges to DPA attacks, in certain respects it also makes the attack easier. Unlike most first-order DPA attacks, the sequential nature of the counter enables the attack to succeed with 
only knowledge of the power measurements. Knowledge of input, output, and initial counter values are not required to implement the attack.

\subsection{Related Work}

Simple power analysis (SPA) attacks have been used to extract portions of keys directly from power traces without requiring knowledge of input messages. Fahn and Pearson used inferential power analysis (IPA), an attack that exploits binary SPA leaks 55. Mayer-Sommer presented attacks exploiting SPA leaks in highamplitude power variations 6]. Mangard presented an SPA attack against the AES key expansion step [7. Messerges et al described SPA attacks on Hamming weight and transition count leaks [8].

Side channel collision attacks were introduced by Dobbertin, and have traditionally targeted SPA leaks using chosen ciphertext 99 [10, 11. Side channel collision attacks can be adapted to the case in which inputs are known to be successive values of a counter.

High-order differential power analysis (HO-DPA) 12 attacks target a hypothesized key-dependent relationship between data parameters in a computation. Previous work has noted that HO-DPA attacks can be applied to situations in which cipher input values are not known 13 .

Fouque and Valette presented the "doubling attack" 14 which exploits the relationship between inputs in successive RSA decryptions to recover the exponent. The attack succeeds despite the fact that the input to the modular exponentiation step is masked by a blinding factor. Messerges presented a second-order DPA attack 15 that defeated a data whitening scheme.

Chari et al [16] and Akkar et al [17] also presented DPA attacks on block ciphers with a "whitening" step.

\section{Preliminaries}

\section{$2.1 \quad$ Notation}

Suppose $X$ and $Y$ are used to denote input and output data of a transformation. (Letters other than $X$ or $Y$ will also be used.) If the transformation is implemented as a sequence of rounds, the input and output of the $i^{\text {th }}$ round are denoted by $X_{i}$ and $Y_{i}$.

Within a round, data may be partitioned into bytes for processing. $X_{i, j}$ and $Y_{i, j}$ denote the $j^{\text {th }}$ bytes of round data $X_{i}$ and $V_{i}$.

$K$ is used to denote input keys, $K_{i}$ denotes the $i^{\text {th }}$ round key derived from $K$, and $K_{i, j}$ denotes the $j^{\text {th }}$ byte of round key $K_{i}$.

\section{Symbols}

The symbol ' $\oplus$ ' denotes the bitwise XOR of two $n$-bit vectors.

The symbol ' + ' denotes the ordinary addition of two numbers.

The symbol 'o' denotes multiplication between two elements of $G F\left(2^{8}\right)$.

The symbol 'I' denotes the concatenation of two vectors. 


\subsection{Description of AES}

Although most readers are no doubt familiar with AES, this section gives a brief review of its design. The round transformations are grouped differently than in the AES standard to facilitate presentation of the attack, but the algorithm described here is equivalent to AES. The review will also familiarize the reader with the notation and concepts used in this paper.

AES is a block cipher that operates on 16-byte blocks of data. It is designed as a sequence of 10,12 , or 14 rounds, depending on whether the key $K$ is 16 , 24 , or 32 bytes in length. The key is expanded by the AES key schedule into 16-byte round keys $K_{i}$.

The round structure of AES encryption. The following transformations are performed during each round of an AES encryption:

1. AddRoundKey

2. SubBytes

3. ShiftRows

4. MixColumn 1 1

These operations are described below, using the following notation for intermediate round states:

$X_{i}$ denotes the input to round $i$ and the AddRoundKey transformation.

$Y_{i}$ denotes the output of the AddRoundKey transformation and the input to the SubBytes transformation.

$Z_{i}$ denotes the output of the SubBytes transformation and the input to the ShiftRows transformation.

$U_{i}$ denotes the output of the ShiftRows transformation and the input to the MixColumns transformation.

$V_{i}$ denotes the output of the MixColumns transformation and the input to the next round: $V_{i}=X_{i+1}$.

AddRoundKey. Each byte of $Y_{i, j}$ is produced by computing the exclusive or (XOR) of a byte of incoming data $X_{i, j}$ with the corresponding byte of round key $K_{i, j}$ :

$$
Y_{i, j}=X_{i, j} \oplus K_{i, j}
$$

SubBytes. Each byte of input data is transformed via an invertible non-linear 8-bit lookup table $S$ :

$$
Z_{i, j}=S\left[Y_{i, j}\right]=S\left[X_{i, j} \oplus K_{i, j}\right] .
$$

${ }^{1}$ The MixColumns operation is not performed in the final round, and an additional AddRoundKey operation is performed after the final round. 
ShiftRows. ShiftRows permutes the bytes within the data vector:

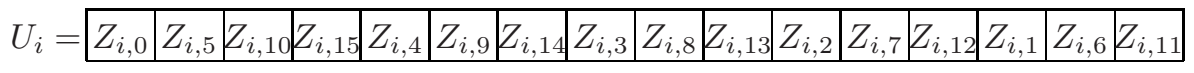

MixColumns. The $j^{\text {th }}$ column of $U_{i}$ is defined to be the four bytes

$$
\left\{U_{i, 4 j}, U_{i, 4 j+1}, U_{i, 4 j+2}, U_{i, 4 j+3}\right\} .
$$

MixColumns is an invertible linear transformation over $G F\left(2^{8}\right)$ performed on the columns of $U_{i}$. The $j^{\text {th }}$ column of output $V_{i}$ is defined to be:

$$
\begin{aligned}
V_{i, 4 j} & =\left(\{02\} \circ U_{i, 4 j}\right) \oplus\left(\{03\} \circ U_{i, 4 j+1}\right) \oplus\left(\{01\} \circ U_{i, 4 j+2}\right) \oplus\left(\{01\} \circ U_{i, 4 j+3}\right) \\
V_{i, 4 j+1} & =\left(\{01\} \circ U_{i, 4 j}\right) \oplus\left(\{02\} \circ U_{i, 4 j+1}\right) \oplus\left(\{03\} \circ U_{i, 4 j+2}\right) \oplus\left(\{01\} \circ U_{i, 4 j+3}\right) \\
V_{i, 4 j+2} & =\left(\{01\} \circ U_{i, 4 j}\right) \oplus\left(\{01\} \circ U_{i, 4 j+1}\right) \oplus\left(\{02\} \circ U_{i, 4 j+2}\right) \oplus\left(\{03\} \circ U_{i, 4 j+3}\right) \\
V_{i, 4 j+3} & =\left(\{03\} \circ U_{i, 4 j}\right) \oplus\left(\{01\} \circ U_{i, 4 j+1}\right) \oplus\left(\{01\} \circ U_{i, 4 j+2}\right) \oplus\left(\{02\} \circ U_{i, 4 j+3}\right)
\end{aligned}
$$

where $\{01\},\{02\},\{03\}$, and $U_{i, 4 j}, U_{i, 4 j+1}, U_{i, 4 j+2}, U_{i, 4 j+3}$ are considered 8-bit vectors representing elements in $G F\left(2^{8}\right)$.

The linearity of the AES MixColumns transformation will be exploited during the attack. Suppose that input data can be selected such that in round $i$, one or more input bytes to the MixColumns operation are unknown, but are known to remain constant across multiple invocations of the AES algorithm. Then the contribution of these constant bytes to $V_{i}$ is equivalent to XORing with fixed constants.

For example, suppose bytes $U_{1,4 j+1}, U_{1,4 j+2}$, and $U_{1,4 j+3}$ are constant (but unknown) across multiple invocations of AES. Then the values

$$
\begin{aligned}
E_{1,4 j} & =\left(\{03\} \circ U_{1,4 j+1}\right) \oplus\left(\{01\} \circ U_{1,4 j+2}\right) \oplus\left(\{01\} \circ U_{1,4 j+3}\right) \\
E_{1,4 j+1} & =\left(\{02\} \circ U_{1,4 j+1}\right) \oplus\left(\{03\} \circ U_{1,4 j+2}\right) \oplus\left(\{01\} \circ U_{1,4 j+3}\right) \\
E_{1,4 j+2} & =\left(\{01\} \circ U_{1,4 j+1}\right) \oplus\left(\{02\} \circ U_{1,4 j+2}\right) \oplus\left(\{03\} \circ U_{1,4 j+3}\right) \\
E_{1,4 j+3} & =\left(\{01\} \circ U_{1,4 j+1}\right) \oplus\left(\{01\} \circ U_{1,4 j+2}\right) \oplus\left(\{02\} \circ U_{1,4 j+3}\right)
\end{aligned}
$$

will be constant, and the MixColumns output can be expressed as

$$
\begin{aligned}
V_{1,4 j} & =\left(\{02\} \circ U_{1,4 j}\right) \oplus E_{1,4 j} \\
V_{1,4 j+1} & =\left(\{01\} \circ U_{1,4 j}\right) \oplus E_{1,4 j+1} \\
V_{1,4 j+2} & =\left(\{01\} \circ U_{1,4 j}\right) \oplus E_{1,4 j+2} \\
V_{1,4 j+3} & =\left(\{03\} \circ U_{1,4 j}\right) \oplus E_{1,4 j+3} .
\end{aligned}
$$

As will be shown in Section 3, the constant, unknown terms $E$ can then be incorporated into the round key of the next round, and effectively ignored.

\subsection{Counter Mode}

Counter mode is a standard mode of operation for block ciphers in which ciphertext is produced by encrypting a counter and XORing the result with the 
plaintext block. Let $B$ be a block cipher using key $K, C$ the initial counter value, and $X_{T}$ the $T^{\text {th }}$ block of plaintext to be encrypted. Then the $T^{\text {th }}$ block of ciphertext $Y_{T}$ is given by

$$
Y_{T}=X_{T} \oplus B_{\text {enc }}(C+T, K) .
$$

Ciphertext is decrypted by XORing it with same encrypted counter value:

$$
X_{T}=Y_{T} \oplus B_{\text {enc }}(C+T, K) .
$$

Since counter values are inputs to the first round only, $C_{j}$ and $T_{j}$ will be used to denote the $j^{\text {th }}$ bytes of $C$ and $T$ respectively, and not their values at round $j$. See 4 for more information on counter mode.

Galois counter mode. Galois counter mode (GCM) [18] is a draft counter mode protocol currently being studied by NIST. In GCM, the initial counter value is derived from a variable-sized initialization vector (IV). If the length of the IV is not exactly 96 bits, then the initial counter value $C$ is derived from the IV using a secret key. In protocols where the IV is exactly 96 bits long, at least part of the initial counter value may be secret. For example, in RFC 4106 [19] the first four bytes of the IV are derived with the AES key and may remain secret. The attack described in this paper assumes that the entire initial counter value $C$ is unknown.

\section{The Attack on AES in Counter Mode}

This section will present a first-order DPA attack against AES in counter mode with unknown initial counter value $C$.

To keep the index notation from getting too cumbersome, the symbol " $T$ " is omitted from subscripts. When data is described as constant or variable, however, it means that the data is constant or variable with respect to $T$. For example, when we say that an attack recovers a variable such as $Z_{1,15}$, it means that it recovers each value the variable took for each value of $T$.

\subsection{Overview}

The main stages of the attack are as follows:

1. Perform data collection.

2. Use DPA against the first round to recover $Z_{1,15}$ and $Z_{1,14}$.

3. Derive the input to the second round, manipulating unknown values symbolically. Eight bytes of input to the second round are unknown constants, but the other eight can be expressed as

$$
X_{2, j}=\tilde{X}_{2, j} \oplus E_{1, j}
$$

where $\tilde{X}_{2, j}$ is known and variable, and $E_{1, j}$ is unknown, but constant. 
4. Use DPA to determine the eight variable bytes of $Z_{2, j}$ corresponding to the 8 variable bytes $X_{2, j}$.

5. Derive the input to the third round, manipulating unknown constants symbolically. Each of the sixteen bytes of $X_{3}$ can be expressed as

$$
X_{3, j}=\tilde{X}_{3, j} \oplus E_{2, j},
$$

where $\tilde{X}_{3, j}$ is known and variable, and $E_{2, j}$ is unknown, but constant.

6. Use DPA to determine the sixteen variable bytes of $Z_{3}$.

7. Derive the input to the fourth round. There are no unknown or constant bytes in $Z_{3}$, so $X_{4}$ can be derived exactly.

8. Perform a standard DPA attack in the fourth round, using known input values $X_{4}$. Iterate the attack into subsequent rounds as necessary, recovering as many round keys as required to reverse the key schedule and obtain the key.

These attack stages are described in detail below.

\subsection{Attack Details}

Step 1: Data Collection. Encrypt $2^{16}$ consecutive blocks of data in counter mode, with unknown initial counter, and initial data blocks given by $X_{1}=$ $C+T$.

Record power traces covering the first four rounds of each encryption. Traces should cover the fifth round as well if the target key is longer than 16 bytes.

Step 2: Recover $Z_{1,15}$. The DPA attack in this step uses the known byte $T_{15}$ as the input, and performs a 15-bit exhaustive search over the bits defined below.

Let $C_{15, l_{o}}$ and $K_{1,15, l_{o}}$ denote the low-order 7 bits of $C_{15}$ and $K_{1,15}$, respectively. Let $C_{15, h i}$ denote the high-order bit of $C_{15}$, and $b_{15}$ denote the XOR of $C_{15, h i}$ with the high-order bit of $K_{1,15}$. Let $\epsilon_{15}$ denote the outgoing carry of $C_{15}+T_{15}$. The reader can verify that

$$
\left(C_{15}+T_{15}\right) \bmod 256=\left(2^{7} * C_{15, h i}\right) \oplus\left(\left(C_{15, l o}+T_{15}\right) \bmod 256\right) .
$$

Then Equations 1 and 4 imply that

$$
\begin{aligned}
Y_{1,15} & =K_{1,15} \oplus\left(\left(C_{15}+T_{15}\right) \bmod 256\right) \\
& =K_{1,15} \oplus\left(2^{7} * C_{15, h i}\right) \oplus\left(\left(C_{15, l_{o}}+T_{15}\right) \bmod 256\right) \\
& =\left(2^{7} * b_{15}\right) \oplus K_{1,15, l o} \oplus\left(\left(C_{15, l_{o}}+T_{15}\right) \bmod 256\right) .
\end{aligned}
$$

Hence, the DPA search only depends on the 15 unknown bits in Equation 5 . the bit $b_{15}$, seven bits of $K_{1,15, l o}$, and seven bits of $C_{15, l_{o}}$. Also, note that the high-order bits of $K_{1,15}$ and $C_{15}$ cannot be distinguished by this search.

The relationship between the parameters is shown in Figure 11. Note that because $Y_{1,15}$ is an eight bit quantity, it does not depend on the carry bit $\epsilon_{15}$. 


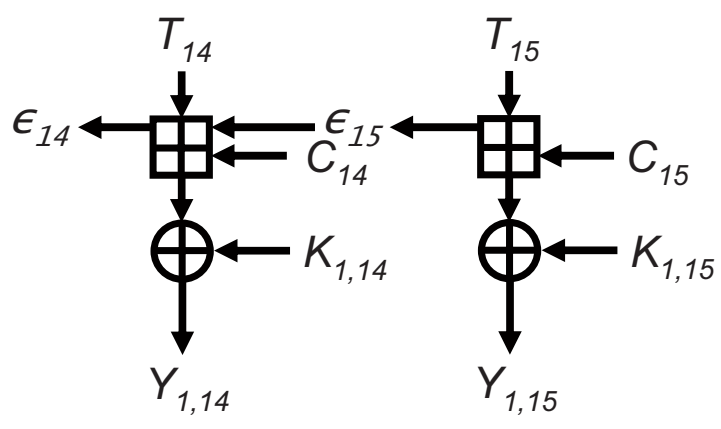

Fig. 1. Relationship between $T, C, K$, and $Y$ for bytes 14 and 15 in Round 1 of the attack

Step 3: Recover $Z_{1,14}$. The DPA attack in this step uses the known byte $T_{14}$ as the input, and performs a 16-bit exhaustive searching over the following bits: the bit $C_{15, h i}$, the low-order 7 bits of $C_{14}$, the low-order 7 bits of $K_{1,14}$, and the bit $b_{14}$, defined as the XOR of the high-order bit of $C_{14}$ with the high-order bit of $K_{1,14}$.

$Y_{1,14}$ is given by

$$
Y_{1,14}=K_{1,14} \oplus\left(\left(C_{14}+T_{14}+\epsilon_{15}\right) \bmod 256\right) .
$$

$Y_{1,14}$ depends on $\epsilon_{15}$, which iteself depends upon $C_{15}$. Hence $C_{15, h i}$ (the only bit of $C_{15}$ not recovered in Step 2) is one of the bits searched for in this step. As in Step 2, the search recovers $b_{14}$ but is unable to distinguish the high-order bits of $K_{1,14}$ and $C_{14}$, nor determine the value of the carry bit $\epsilon_{14}$.

Step 4: Select those values of $T, 0 \leq T<2^{16}$ for which the bytes $X_{1,0} \ldots X_{1,13}$ remain constant.

These bytes will remain constant if the carry bit $\epsilon_{14}$ remains constant. Let $\left(C_{14, l o} \| C_{15}\right)$ denote the 15 -bit integer resulting from the concatenation of $C_{14, l o}$ and $C_{15}$. Even though the actual value of $\epsilon_{14}$ is unknown, the reader can verify that it remains constant for those values of $\mathrm{T}$ satisfying

$$
2^{15}-\left(C_{14, l o} \| C_{15}\right) \leq T<2^{16}-\left(C_{14, l o} \| C_{15}\right) .
$$

This gives a subset of $T$ values for which, after applying the AddRoundKey transformation to $X_{1}$ and SubBytes transformation to $Y_{1}$ :

- The 14 bytes $\left\{Z_{1,0} \ldots Z_{1,13}\right\}$ are unknown, but constant.

- The bytes $Z_{1,14}$ and $Z_{1,15}$ are known, and varying.

The remainder of the attack proceeds using only the $2^{15}$ traces corresponding to this subset of $T$ values. 
Step 5: Apply the ShiftRows and MixColumns operation to $Z_{1}$ to compute $V_{1}=X_{2}$, the input to Round 2 , manipulating unknown values symbolically.

Using Equation 3 (discussed in $\$ 2.2$ ), it can be shown that $X_{2}$ has the following properties:

- Bytes $X_{2,0} \ldots X_{2,7}$ have the form

$$
X_{2, j}=\tilde{X}_{2, j} \oplus E_{1, j}
$$

where $\tilde{X}_{2, j}$ are known and vary with $T$, and the $E_{1, j}$ are unknown, but constant with respect to $\mathrm{T}$.

- Bytes $X_{2,8} \ldots X_{2,15}$ are unknown, but constant.

Step 6: Apply the Round 2 AddRoundKey transformation to $X_{2}$ to compute $Y_{2}$, manipulating unknown values symbolically.

For $X_{2,0} \ldots X_{2,7}$, the results are

$$
\begin{aligned}
Y_{2, j} & =\left(\tilde{X}_{2, j} \oplus E_{1, j}\right) \oplus K_{2, j} \\
& =\tilde{X}_{2, j} \oplus\left(E_{1, j} \oplus K_{2, j}\right) \\
& =\tilde{X}_{2, j} \oplus \tilde{K}_{2, j} .
\end{aligned}
$$

For these bytes, the AddRoundKey transformation is equivalent to XORing known and varying input data $\tilde{X}_{2, j}$ with constant "key" bytes $\tilde{K}_{2, j}$.

Step 7: Use DPA to recover $\tilde{K}_{2,0} \ldots \tilde{K}_{2,7}$ using $\tilde{X}_{2,0} \ldots \tilde{X}_{2,7}$ as known inputs into the relationship:

$$
Z_{2, j}=S\left[\tilde{X}_{2, j} \oplus \tilde{K}_{2, j}\right]
$$

This step displays one of the most crucial (and interesting) features of the attack. We cannot use the correct values for $X_{2, j}$ as input to the DPA attack, since they are unknown. Instead, we treat the known values $\tilde{X}_{2, j}$ as the input. They differ from the correct values by fixed error terms $E_{1, j}$. The keys recovered are not the correct keys, but differ from them by the same fixed error terms. As Equations 8 and 9 show, these error terms then cancel when $Y_{2, j}$ and $Z_{2, j}$ are computed, leaving us with the correct values for them.

Since bytes $X_{2,8} \ldots X_{2,15}$ are unknown but constant, the corresponding bytes $Z_{2,8} \ldots Z_{2,15}$ are also unknown, but constant.

At the end of this step,

$-Z_{2,0} \ldots Z_{2,7}$ are varying, and known exactly.

- $Z_{2,8} \ldots Z_{2,15}$ are unknown, but constant.

Step 8: As in step 5, apply the ShiftRows and MixColumns operation to $Z_{2}$ to compute $V_{2}=X_{3}$, the input to Round 3 , manipulating unknown values symbolically. 
Again, the result can be expressed in terms of a known vector $\tilde{X}_{3}$ as:

$$
X_{3}=\tilde{X}_{3} \oplus E_{2},
$$

where

- $E_{2}$ is a vector of 16 unknown, but constant bytes.

- Every byte of $\tilde{X}_{3}$ is known and variable.

Step 9: Use DPA to recover $Z_{3}$.

The attack uses $\tilde{X}_{3}$ as the known variable input, and recovers $\tilde{K}_{3}$, and all 16 correct bytes of $Z_{3}$.

Step 10: Given all 16 correct values of $Z_{3}$, apply the ShiftRows and MixCols operation to obtain $V_{3}=X_{4}$.

Note that at this point all the error terms are gone, and $X_{4}$ is the correct input to round 4 .

Step 11: Use DPA to obtain $K_{4}$ using the known, variable Round 4 input $X_{4}$.

If 24 or 32-byte keys are used, repeat Steps 10 and 11 in Round 5 to recover another round key.

Step 12: After recovering enough round keys, reverse the key schedule and determine the original AES key.

\section{Results}

The attack was implemented against a smart card performing AES-128 in counter mode with unknown initial counter value.

Step 1: Power traces were collected during $2^{16}$ sequential encryptions.

Step 2: A custom program was written to search over $K_{1,15, l o}, C_{15, l o}$, and $b_{15}$, using the input values of $T_{15}$ to generate predicted values of $Z_{1,15}$. Then DPA was used to evaluate the predictions. The analysis took about 11 minutes on a Dell workstation and revealed that $\left(K_{1,15, l o}, C_{15, l o}, b_{15}\right)=(30 \mathrm{~h}, 42 \mathrm{~h}, 0)$.

Because evaluating $2^{15}$ difference traces individually is somewhat tedious, we measured and plotted the average square of the total amplitude of the differentials observed in each differential trace.

In addition to the primary spike at $(30 \mathrm{~h}, 42 \mathrm{~h}, 0)$, this representation reveals secondary harmonics at $(70 \mathrm{~h}, 02 \mathrm{~h}, 1),(10 \mathrm{~h}, 62 \mathrm{~h}, 0)$, and a few other locations as expected. Note that the AES substitution table is extremely flat, and does not contribute these harmonic peaks. Rather the spikes observed in this figure 


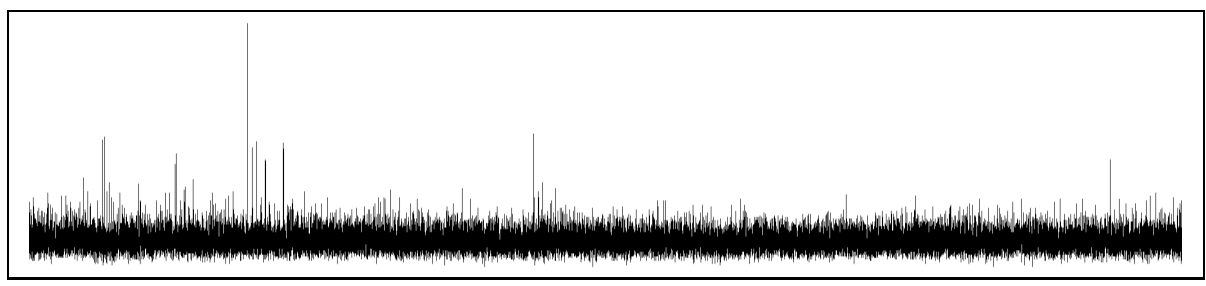

Fig. 2. DPA search results in compact form, showing primary spike for $K_{1,15, l o}=30 \mathrm{~h}$, $C_{15, l_{o}}=42 \mathrm{~h}$, and $b_{15}=0$ at offset 6210 of 32678

are due to relationships between the input parameters, stemming from the structure of the XOR and ADD combination 2

Step 3: A standard DPA attack was implemented to recover $C_{15, h i}, K_{1,14, l o}$, $C_{14, l o}$, and $b_{14}$, using $T_{14}$ as the primary input, and $T_{15}$ and $C_{15, l o}$ to derive the $\epsilon_{15}$. This information was then used to compute $Z_{1,14}$.

The analysis revealed that $C_{15, h i}=0, K_{1,14, l o}=65 \mathrm{~h}, C_{14, l o}=35 \mathrm{~h}$, and $b_{14}=0$.

Step 4: The recovered values

$$
\left(C_{14, l o}, C_{15}\right)=(35 \mathrm{~h}, 42 \mathrm{~h})
$$

were used to determine the values of $T$ for which bytes $0 . .13$ of $C+T$ remain constant. These values are given by

$$
2^{15}-3542 \mathrm{~h} \leq T<2^{16}-3542 \mathrm{~h} \quad \Rightarrow \quad 19134 \leq T<51902 .
$$

For the remainder of the attack, only those power traces for which $T$ is in this range were used 3

Step 5: In this step we need to apply the ShiftRows and MixColumns to the $Z_{1}$ to compute $X_{2}$. Only $Z_{1,14}$ and $Z_{1,15}$ are known, however, and our analysis software is not configured to handle variables symbolically. As noted in Step 7, however, the DPA attack to recover the eight bytes $Z_{2,0} \ldots Z_{2,7}$ is unaffected

${ }^{2}$ Consider the eight-bit construction $Y=f(K, C, X)=K \oplus(C+X) \bmod 256$. There are fifteen "harmonic" values of $\left(K_{i}, C_{i}\right)$ for which $f\left(K_{i}, C_{i}, X\right)=f(30 \mathrm{~h}, 42 \mathrm{~h}, X)$ for half of the possible values of $X$. With these related keys the input to the SubBytes would be correct exactly $50 \%$ of the time. At the output of the SubBytes operation, individual bits of $Z_{1,15}$ are correct about $75 \%$ of the time, leading to high-amplitude spikes in single-bit analysis. The Hamming weight of $Z_{1,15}$ is correct $57 \%-64 \%$ of the time for these related keys. If correlation or other multi-bit analysis methods are used the statistical significance of the harmonic spikes will be observed with greater clarity.

3 The signal-to-noise levels observed in this dataset were sufficiently high that we actually performed the attack on round two using only $2^{13}$ traces. 
by the actual values of the error terms $E_{1, j}$ derived from the unknown bytes of $Z_{1}$. Hence, to complete this step, we substituted the value 0 for each unknown constant byte. We were then able to perform the ShiftRows and MixColumns transformations using our existing software.

Steps 6,7: DPA was used to recover "key" bytes

$$
\tilde{K}_{2,0}=K_{2,0} \oplus E_{1,0} \quad \ldots \quad \tilde{K}_{2,0}=K_{2,7} \oplus E_{1,7},
$$

using input data

$$
\tilde{X}_{2,0}=X_{2,0} \oplus E_{1,0} \quad \ldots \quad \tilde{X}_{2,7}=X_{2,7} \oplus E_{1,7} .
$$

The 8 bytes of $\tilde{K}_{2,0} \ldots \tilde{K}_{2,7}$ recovered were:

$$
\tilde{\mathrm{K}}_{2}=\text { 30451E9FD1923450--------------h }
$$

Given the $\tilde{K}_{2, j}$ and $\tilde{X}_{2, j}$ we calculated the correct values $Z_{2,0} \ldots Z_{2,7}$ by:

$$
Z_{2, j}=S\left[\tilde{X}_{2, j} \oplus \tilde{K}_{2, j}\right]
$$

Step 8: At this point, bytes $Z_{2,0} \ldots Z_{2,7}$ are known and variable, and the remaining $Z_{2, j}$ are unknown but constant. As in Step 5, the unknown $Z_{2, j}$ are set to zero, and the second round completed. All 16 bytes of $V_{2}=X_{3}$ have the form

$$
X_{3, j}=\tilde{X}_{3, j} \oplus E_{2, j}
$$

where the $\tilde{X}_{3, j}$ is known and variable, and $\tilde{E}_{2, j}$ are unknown but constant.

Step 9: As in Step 7, the $\tilde{X}_{3, j}$ were used as known input to a DPA attack to recover $\tilde{K}_{3, j}$ and $Z_{3, j}$. All 16 bytes of $Z_{3}$ were recovered, as was the entire key $\tilde{K}_{3}$.

$$
\tilde{\mathrm{K}}_{3}=7 \mathrm{~A} 610872 \mathrm{DE} 8 \mathrm{FE} 299708 \mathrm{~A} 89 \mathrm{~A} 85 \mathrm{DD} 9914 \mathrm{Dh}
$$

Step 10: With all 16 values of $Z_{3}$ known, we simply completed the round to compute $V_{3}=X_{4}$, the correct input to Round 4 .

Step 11: We performed standard DPA using correct, known variable inputs $X_{4}$ The following key was recovered:

$$
\mathrm{K}_{4}=\text { 52438AAD476E016D31EAE1CDAE8EOF3Dh }
$$

Step 12: Since the target of this attack was performing AES-128, at this point we had sufficient material from the key schedule to compute the correct input key. Running the key schedule backwards gave:

$$
\begin{aligned}
& \mathrm{K}_{4}=52438 A A D 476 E 016 D 31 \mathrm{EAE} 1 \mathrm{CDAE} 8 \mathrm{E} 0 \mathrm{~F} 3 \mathrm{Dh} \\
& \mathrm{K}_{3}=156 \mathrm{~B} 0676152 \mathrm{D} 8 \mathrm{BC07684E0A09F64EEF0h} \\
& \mathrm{K}_{2}=\mathrm{F} 6 \mathrm{C} 0556800468 \mathrm{DB} 663 \mathrm{~A} 96 \mathrm{~B} 60 \mathrm{E} 9 \mathrm{E} 00 \mathrm{E} 5 \mathrm{~h} \\
& \mathrm{~K}_{1}=\text { CC8D5116F686D8DE63EFE6D68A496530h }
\end{aligned}
$$


Therefore, the 128-bit AES key recovered by this attack is equal to

$$
\mathbf{K}=\text { CC8D5116F686D8DE63EFE6D68A496530h }
$$

and the attack is complete.

\section{Concluding Remarks}

In this paper we described a first-order DPA attack against AES in counter mode with an unknown counter. We introduced a technique to shift unknown constant data onto round keys such that they could be effectively ignored. This compensates for the unknown counter value, as well as the counter mode property that only the low-order bytes of the input change.

The techniques presented here were used to mount an attack against a smart card implementation of AES in counter mode. The attack required only $2^{13}$ traces pulled from a set of $2^{16}$ sequential operations. The same technique might still succeed using $2^{8}$ or fewer sequential traces, if the leakage rates are sufficiently high.

Countermeasures that defend against first-order DPA attacks should be effective against this attack as well. Also, modifying the method by which the counter updates (using a linear feedback shift register, for example) would present a challenge to this attack.

The techniques in this paper can be applied to other cryptographic algorithms. In general, when an unknown constant is mixed with known variable data, DPA can be used to mount an attack if the mixing function is nonlinear. As we have shown, if the mixing function is linear, evaluation of the secret constant can often be postponed until an attack is possible.

\section{References}

1. Kocher, P., Jaffe, J., Jun, B.: Differential Power Analysis. In: Wiener, M.J. (ed.) CRYPTO 1999. LNCS, vol. 1666, pp. 388-397. Springer, Heidelberg (1999)

2. Chari, S., Jutla, C.S., Rao, J.R., Rohatgi, P.: Towards Sound Approaches to Counteract Power-Analysis Attacks. In: Wiener, M.J. (ed.) CRYPTO 1999. LNCS, vol. 1666, pp. 398-412. Springer, Heidelberg (1999)

3. National Institute of Standards and Technology: Advanced Encryption Standard (AES) (FIPS PUB 197). National Institute of Standards and Technology. Federal Information Processing Standards Publication 197 (FIPS 197) (November 2001)

4. Dworkin, M.: Recommendation for Block Cipher Modes of Operation:Methods and Techniques National Institute of Standards and Technology. Special Publication 800-38A (December 2001)

5. Fahn, P.N., Pearson, P.K.: IPA: A New Class of Power Attacks. In: Koç, Ç.K., Paar, C. (eds.) CHES 1999. LNCS, vol. 1717, pp. 173-186. Springer, Heidelberg (1999)

6. Mayer-Sommer, R.: Smartly Analyzing the Simplicity and the Power of Simple Power Analysis on Smartcards. In: Paar, C., Koç, Ç.K. (eds.) CHES 2000. LNCS, vol. 1965, pp. 78-92. Springer, Heidelberg (2000) 
7. Mangard, S.: A Simple Power-Analysis (SPA) Attack on Implementations of the AES Key Expansion. In: Lee, P.J., Lim, C.H. (eds.) ICISC 2002. LNCS, vol. 2587, pp. 343-358. Springer, Heidelberg (2003)

8. Messerges, T., Dabbish, E., Sloan, R.: Investigations of Power Analysis Attacks on Smartcards. In: Proc. USENIX Workshop on Smartcard Technology, pp. 151-162 (1999)

9. Ledig, H., Muller, F., Valette, F.: Enhancing Collision Attacks. In: Joye, M., Quisquater, J.-J. (eds.) CHES 2004. LNCS, vol. 3156, pp. 176-190. Springer, Heidelberg (2004)

10. Schramm, K., Wollinger, T., Paar, C.: A New Class of Collision Attacks and its Application to DES. In: Johansson, T. (ed.) FSE 2003. LNCS, vol. 2887, pp. 206222. Springer, Heidelberg (2003)

11. Schramm, K., Leander, G., Felke, P., Paar, C.: A Collision-Attack on AES Combining Side Channel- and Differential- Attack. In: Joye, M., Quisquater, J.-J. (eds.) CHES 2004. LNCS, vol. 3156, pp. 163-175. Springer, Heidelberg (2004)

12. Kocher, P., Jaffe, J., Jun, B.: Introduction to Differential Power Analysis and Related Attacks (Technical Report) (1998),

http://cryptography.com/resources/whitepapers/DPATechInfo.pdf
http://web.archive.org/web/19990504025809/www.cryptography.com/dpa/
technical/index.html
http://tinyurl.com/244azs http://tinyurl.com/2zgfc3

13. Jaffe, J., Jun, B., Kocher, P.: Advanced Topics 1. In: Presentation for the DPA Workshop, Chicago IL, Cryptography Research (May 14-15, 1999)

14. Fouque, P.-A., Valette, F.: The Doubling Attack - Why Upwards Is Better than Downwards. In: D.Walter, C., Koç, Ç.K., Paar, C. (eds.) CHES 2003. LNCS, vol. 2779, pp. 269-280. Springer, Heidelberg (2003)

15. Messerges, T.: Using Second-Order Power Analysis to Attack DPA Resistant Software. In: Paar, C., Koç, Ç.K. (eds.) CHES 2000. LNCS, vol. 1965, pp. 238-251. Springer, Heidelberg (2000)

16. Chari, S., Jutla, C., Rao, J.R., Rohatgi, P.: A Cautionary Note Regarding Evaluation of AES Candidates on Smart-Cards AES Second Candidate Conference (February 1999), http://csrc.nist.gov/encryption/aes/round1/conf2/papers/chari.pdf

17. Akkar, M.-L., Bevan, R., Dischamp, P., Moyart, D.: Power Analysis, What Is Now Possible. In: Okamoto, T. (ed.) ASIACRYPT 2000. LNCS, vol. 1976, pp. 489-502. Springer, Heidelberg (2000)

18. McGrew, D.A., Viega, J.: The Galois/Counter Mode of Operation (GCM) National Institute of Standards and Technology. Draft Special Publication 800-38D (May 31, 2005)

19. Viega, J., McGrew, D.: The Use of Galois/Counter Mode (GCM) in IPsec Encapsulating Security Payload (ESP), RFC 4106 (June 2005) 